\title{
Effect of particle size distribution on the correlation between liquefaction resistance and shear wave velocity of granular soils
}

\author{
Changtao Zhou ${ }^{1,}{ }^{*}$, Xiao Min Xu ${ }^{2, \dagger}$, and Yi Pik Cheng ${ }^{1, \ddagger}$ \\ ${ }^{1}$ Department of Civil, Environmental and Geomatic Engineering University College London, London, UK \\ 2 Department of Engineering, University of Cambridge, Cambridge, UK
}

\begin{abstract}
Shear wave method has been increasingly popular in assessing the liquefaction potential of granular soils. Two particle-scale parameters, the inter-particle friction and the shear modulus of grains, play vital roles in correlation between Cyclic Resistance Ratio $(C R R)$ and shear wave velocity corrected by overburden stress $\left(V_{s l}\right)$. Series of drained one-dimensional compression tests were simulated on samples of different inter-particle friction angles assigned during preparation stage. Uniformity coefficients of these Particle Size Distribution (PSD) curves are 2 and 4 whose average particle size $d_{50}$ are identical. The shearing results, as well as their assigned inter-particle friction angles form calibration curves for real sands. Dissimilar PSD curves result in different calibration outcomes. For Silica sand no.8, these curves give divergent interparticle friction angles. This study calibrates particle shear modulus for Silica sand no.8 as well. Different PSD curves give divergent values of particle shear modulus. PSDs show impacts on calibrations of both vital parameters, which have converse effects on $C R R-V_{s I}$ curves. This study suggests that the $C R R-V_{s I}$ correlation should be independent of PSDs.
\end{abstract}

\section{Introduction}

Soil liquefaction is one of the hardest challenges that scientists and engineers are facing. Shear wave velocity corrected for overburden stress $\left(V_{s l}, \mathrm{~s}\right.$ for shear and 1 for principal direction of overburden stress) is widely considered as a promising alternative to assess the liquefaction resistance of granular soils. Previous researchers[1-3] performed numbers of cyclic tri-axial and dynamic centrifuge tests, whose work verified the reliability and suggested the $C R R-V_{s l}$ correlation could be affected by soil types because the shear modulus $\left(G_{g}\right)$ and the inter-particle friction parameter $\left(\mu_{g}\right)$ of soil particles play vital roles[4], as shown by equation (1).

$$
\begin{gathered}
C R R=\alpha_{0} /\left(G_{g} / \mathrm{G}_{\mathrm{g} 0}\right)^{\mathrm{m} \beta} \cdot\left[\rho_{\mathrm{g}}\left(V_{s I}\right)^{2} / \mathrm{P}_{\mathrm{a}}\right]^{\beta / 2} \\
\beta=\mathrm{B} \cdot \arctan \left(\mathrm{A} \cdot \mu_{g}\right)+\mathrm{C}
\end{gathered}
$$

where $\mathrm{G}_{\mathrm{g} 0}=1 \mathrm{GPa}, \alpha_{0}=7.218 \times 10^{-8}, \mathrm{~m}=0.32, \mathrm{~A}=4.890$, $\mathrm{B}=0.971$, and $\mathrm{C}=4.191 . \mathrm{P}_{\mathrm{a}}$ is the atmospheric pressure. $\rho_{\mathrm{g}}$ is the density of grains.

It is difficult to directly measure the inter-particle friction parameter or the shear modulus of grains. By numerical tests, $\mathrm{Xu}$ et al. (2015) calibrated them for Silico Sand no.8. The value of $\varphi_{g}$ obtained is $39.9^{\circ}$, and $G_{g}$ is $6.649 \mathrm{GPa}[4,5]$. Using these two calibrated parameters, they produced a prediction curve which achieved $92 \%$ accuracy in separating liquefied and non-liquefied cases of centrifuge tests.

\footnotetext{
* Changtao Zhou: changtao.zhou.14@alumni.ucl.ac.uk

$\dagger$ Xiaomin Xu: xx787@cam.ac.uk

$\$$ Yi Pik Cheng: yi.cheng@ucl.ac.uk
}

Note the calibration of micro mechanical properties is based on the DEM soil modelling. The PSD could have impacts on the calibration outcomes, which indirectly would affect prediction curves. DEM modelling enables us to modify micro parameters of particles, and see how they would affect the outcomes of macro tests. The increment of $\mu_{g}$ results in higher degree of induced structural anisotropy and a higher rates of dilation in shearing tests[6, 7]. The initial fabric of soil principally affects mechanical behaviours in numerical modelling, which can explain the experimental behaviour of sands [8]. Samples of different PSDs have dissimilar initial contacts fabric inside. Many novel laboratory tests show the PSDs have impacts on soil sample's peak friction angles[9, 10].

Particles' shear modulus could be calibrated by comparing numerical and laboratory shearing wave tests, based on the assumption that the real sand follows the same $V_{s^{-}} G_{g}$ power law[4]. PSD of samples could affect the outcome of $G_{g}$ because a sample of lower void ratio tends to have a higher shear wave velocity inside[11], and a better graded assembly would achieve higher initial bulk density at any relative density level.

The objective of this paper is to show how and why PSDs affect calibrations for Silico sand no.8, in terms of the inter-particle friction and the shear modulus, and eventually the prediction curve for liquefaction resistance, in that the previous calibration[4] employed only one single uniform PSD curve. This paper conducted an integrated analysis on what PSD effects are. 


\section{Methodology}

\subsection{Platform and Parallel Processing}

The DEM simulations were performed on the PFC3D (Particle Flow Code in 3 dimensions) version 3.00 and version 3.10. The author developed codes based on PFC3Dv3.10, which can parallel-process assemblies and shearing tests.

Table.1 Particles numbers within samples

\begin{tabular}{lcr}
\hline PSD curve & $C u$ & Particles \\
\hline C2 & 2.00 & 43,764 \\
C4 & 4.00 & 141,344 \\
Xu et al. (2015) & 1.17 & 28,335 \\
\hline
\end{tabular}

Table 1 shows the particles numbers inside samples of PSD curves in this study (C2\&C4), as well as previous study[4]. The limit of one single processor is of the order of 100,000 particles in three dimensions, in terms of the computation speed[12]. Performing particle generation and loading for PSD curve C4 could take extremely longer time. Parallel-supportive codes make advantage of powerful multiple-core processors. The assembly of particles is divided up spatially into several contiguous regions, and each processor takes charge of one region. In Fig. 2, particles are coloured according to their processors. Data of each region are stored locally in the corresponding processor's memory. Communication occurs merely for exchanges across interfaces between regions, in standard local network. Fig. 1 shows the time for the generation of increasing numbers of particles with different number of multi processors. Multi processors appear a significant efficiency in large-scale computation.

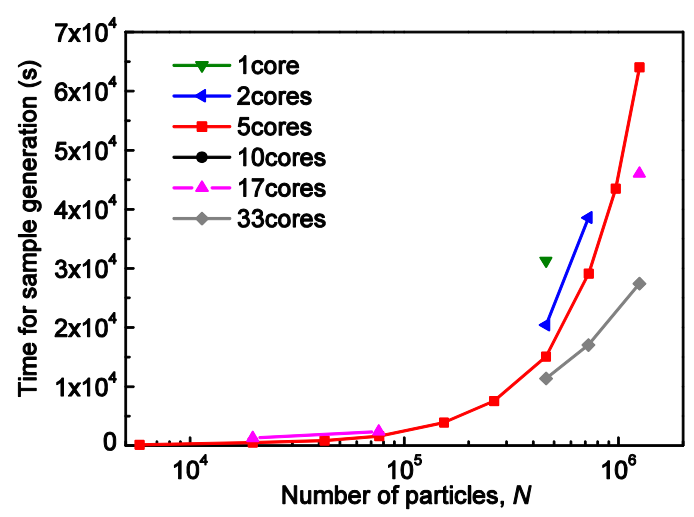

Fig. 1. Time needed for assemblies generation containing different numbers of particles

This paper imitates the practical tri-axial tests by making cylindrical assemblies. Samples were divided up along their long dimension, so that the exchanges of information between regions are kept to a minimum[12]. Walls were created on all processors. Tests' procedure remained the same as those single-processed ones. Test values were recorded locally by each processor, then accumulated by rejecting overlaps of shared balls at interfaces.

\subsection{Sample preparation}

Fig. 2 shows both PSD curves share the identical $\mathrm{d}_{50}$, $0.175 \mathrm{~mm}$. Aspect ratio of height to diameter maintained 2.0. $\mathrm{D} / \mathrm{d}_{50}=20$ is the smallest size that a DEM specimen gives a converged and smooth results[5].

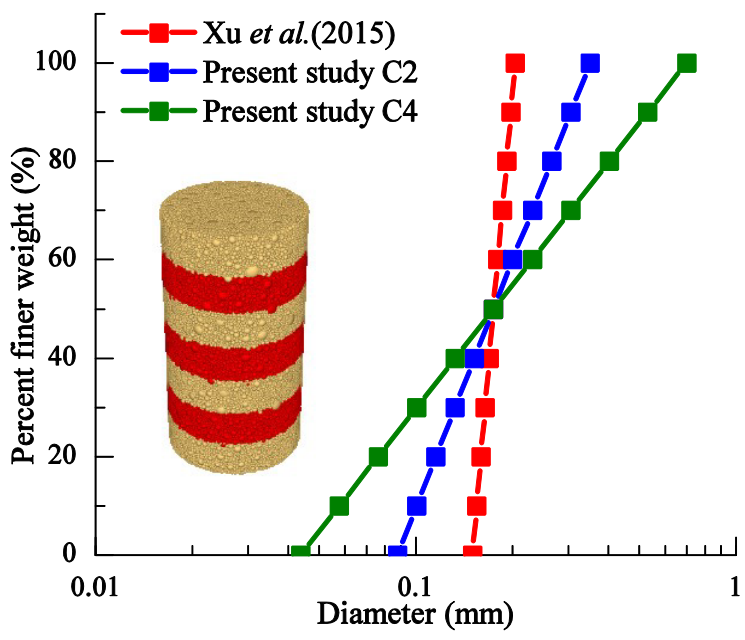

Fig. 2. PSD curves employed and a visualisation of the densest sample of $\mathrm{C} 4$

At beginning, particles were generated by 10 groups from bigger to smaller, randomly in a cylindrical region ( $4 \mathrm{~mm}$ wide, $8 \mathrm{~mm}$ high) constructed by rigid frictionless walls. Particles in one group, spheres of the same radius, were generated as smaller balls then expanded. The HertzMindlin contact model was used due to its suitability for pressure-dependent behaviourr at small strain[4, 12]. Gravity was neglected. Each particle was prescribed with radius, shear stiffness $\left(\mathrm{G}_{\mathrm{g}}=1 \mathrm{GPa}\right)$, Poisson ratio $\left(\mathrm{v}_{\mathrm{g}}=0.2\right)$, density $\left(\rho_{\mathrm{g}}=2630 \mathrm{~kg} / \mathrm{m}^{3}\right)$, and inter-particle friction $\left(\mu_{g}\right)$.

Then the assemblies were consolidated to an isotropic stress state. Note the initial void ratio $\left(e_{0}\right)$ of this paper is defined after a specimen is iso-compressed. Loading was implanted by a servo-control mechanism written for the Hertz-Mindlin contact model. The $e_{0}$ of a specimen could be affected by the porosity input (void/bulk), ban or allowance of spinning, and the friction parameters in generation. Friction of particles was switched to the desired ones when isotropic stresses reached $90 \%$ of the required value and remained the same to the equilibrium state (the mean unbalanced force over the mean contact force $\bar{f}^{u} / \bar{f}^{c} \leq 10^{-3}$ ) at the desired stress.

Samples' generation follows a trial and error manner[4, $6,13]$, to find the maximum and the minimum void ratios $\left(\mathrm{e}_{\max }, \mathrm{e}_{\min }\right)$ at the required stress state. For each PSD curve, the $\mathrm{e}_{\max }$ is reached by setting friction parameter higher and banning particles spinning at generation stage, while the $\mathrm{e}_{\min }$ is achieved by freeing the spinning and setting particles frictionless. The trial of initial parameter falls to the porosity setting at the beginning of generation. The relative density $\left(D_{r}\right)$ of the DEM specimens is defined as $\left(\mathrm{e}_{\max }-e\right) /\left(\mathrm{e}_{\max }-\mathrm{e}_{\min }\right)$, where $e$ is the void ratio of each one.

Samples of PSD curve C2 have void ratios ranging $0.481-0.694$. These of $\mathrm{C} 4$ have void ratio range of 0.338 0.519 .

\subsection{Drained strain-controlled tri-axial tests}

The densest sample (of $\mathrm{e}_{\mathrm{min}}$ ) of each PSD curve was used to perform tri-axial shearing tests. 
Densest specimens were assigned with different interparticle friction parameters (range from 0.1 to 0.5 ). Every time-step, the servo-control function adjusts the radial velocity of the cylindrical wall, according to differences between the actual stress acting on and the required pressure, to keep the confinement pressure at $100 \mathrm{kPa}$ during shearing. The strain rate was checked by using the inertial number defined as $I=\dot{\varepsilon} d \sqrt{\rho / p^{\prime}}$, where $\dot{\varepsilon}$ is the axial-loading rate, $\rho$ is the grain density and $p^{\prime}$ is the mean effective stress. It kept the inertia $I \leq 2.5 e 10^{-3}$ to ensure the quasi-steady conditions[14].

The axial and radical stresses are recorded by dividing the accumulated contact forces on walls by their area. The maximum internal friction angle $\left(\varphi_{\max }\right)$ was obtained by finding the highest ratio of the deviator stress $(q)$ over the mean effective stress $\left(p^{\prime}\right)$. The correlation of $\varphi_{\max }-\varphi_{g}$ is established by aligning the maximum internal friction angles $\left(\varphi_{\max }\right)$ with the inter particle friction angles $\left(\varphi_{g}\right)$. In this study we call this correlation $\varphi_{g}$ calibration curves.

$$
\sin (\varphi)=3\left(q / p^{\prime}\right) /\left(6+q / p^{\prime}\right)=3\left(\sigma_{1}-\sigma_{3}\right) /\left(5 \sigma_{1}+7 \sigma_{3}\right)
$$

\subsection{Shear wave velocity measurements}

This study follows the method of previous research[4] to calibrate grains' shear modulus. $G_{g}$ was calibrated based on the linear $V_{s}-D_{r}$ relationship. For each PSD curve, samples of different relative density $\left(D_{r}\right)$ were tested for wave propagation velocity $\left(V_{s}\right)$ inside. It is well known that the value of small strain shear modulus $\left(G_{\max }\right)$ could be obtained from the shear wave velocity $\left(V_{s}\right)$.

$$
G_{\max }=\rho_{\mathrm{b}} V_{s}^{2}
$$

where $\rho_{b}$ is the bulk density of tested samples.

This study uses equation (3) reversely to get $V_{s}$ of tested samples. Assemblies were rested to a state that velocities of particles inside are lower than $10^{-3} \mathrm{~m} / \mathrm{s}$. Then specimens were subjected to axial loading to a very small strain $\left(<10^{-6}\right)$, with stress state remaining $100 \mathrm{kPa}$ and isotropic. It gives the dynamic shear modulus $\left(G_{\max }\right)$, with which equation (3) could calculate an equivalent $V_{s}$.

\section{Results and observations}

\subsection{Inter-particle friction calibration}

All these $\varphi_{\max }-\varphi_{g}$ relationships for DEM assemblies used could be expressed as equation (4)

$$
\varphi_{\max }=\mathrm{a}\left(\varphi_{g}\right)^{\mathrm{b}}
$$

Table.2 Calibration results of Inter-particle friction angle by using various PSD curves

\begin{tabular}{lcccr}
\hline PSD curve & $\mathrm{a}$ & $\mathrm{b}$ & $\mathrm{R}^{2}$ & $\varphi_{g}\left(^{\circ}\right)$ \\
\hline $\mathrm{C} 2$ & 11.524 & 0.312 & 0.999 & $\mathbf{3 3 . 3}$ \\
$\mathrm{C} 4$ & 10.812 & 0.356 & 0.999 & $\mathbf{2 5 . 7}$ \\
Xu et al. (2015) & 10.121 & 0.332 & $/$ & $\mathbf{3 9 . 9}$ \\
\hline
\end{tabular}

Fig. 3 presents these $\varphi_{\max }-\varphi_{g}$ relationships obtained from shearing tests based on PSD curves C2 and C4. Sets of $\mathrm{a}, \mathrm{b}$ was obtained as presented in Table 2, which also gives their relative coefficients. The calibrated inter- particle friction angle $\left(\varphi_{g}\right)$ for Silico sand no.8 could be determined by both $\varphi_{\max }-\varphi_{g}$ relationships. The right-hand column of Table 2 presents the calibration results based on both calibration curves, and the previous study[4] as well. It shows that the better gradation, the smaller calibrated $\mu_{g}$ value.

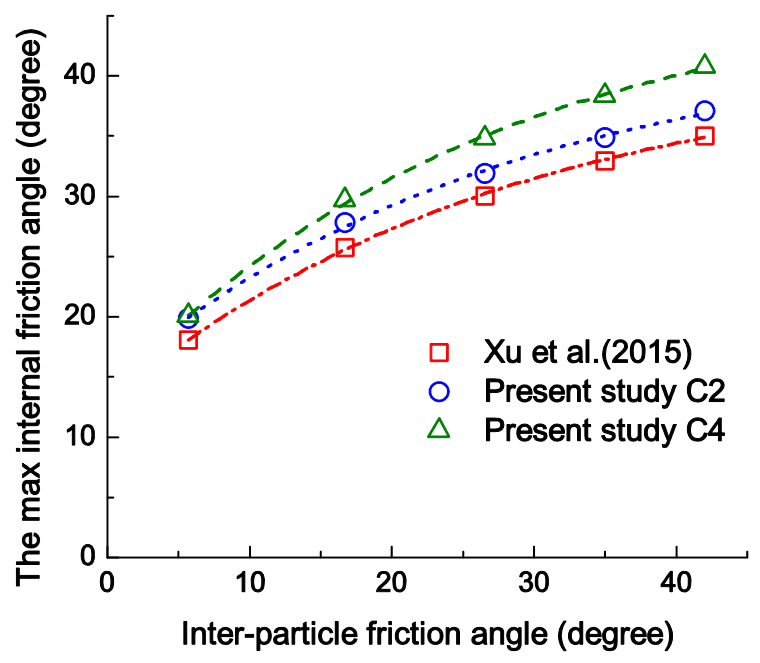

Fig. 3. $\varphi_{g}$ calibration curves of PSD curves

\subsection{Grains' shear modulus calibration}

Specimens of different $D_{r}$ were assigned with a same value of $\mu_{g}, 0.5$. Based on the assumption that real sand follows the same $V_{s^{-}} G_{g}$ power law, the particle shear modulus $\left(G_{g}\right)$ could be calibrated by comparing values of $V_{s}$ obtained from both lab tests and the equivalent shear wave velocity tests at similar relative density range[4, 5].

$$
V_{s}=\alpha^{\prime}\left(G_{g}\right)^{\beta^{\prime}}
$$

where $\alpha^{\prime}=94.9813$, and $\beta^{\prime}=0.3204$.

As shown in Fig. 4, shear wave velocity tests produced various $V_{s}-D_{r}$ relationships for different PSD curves.

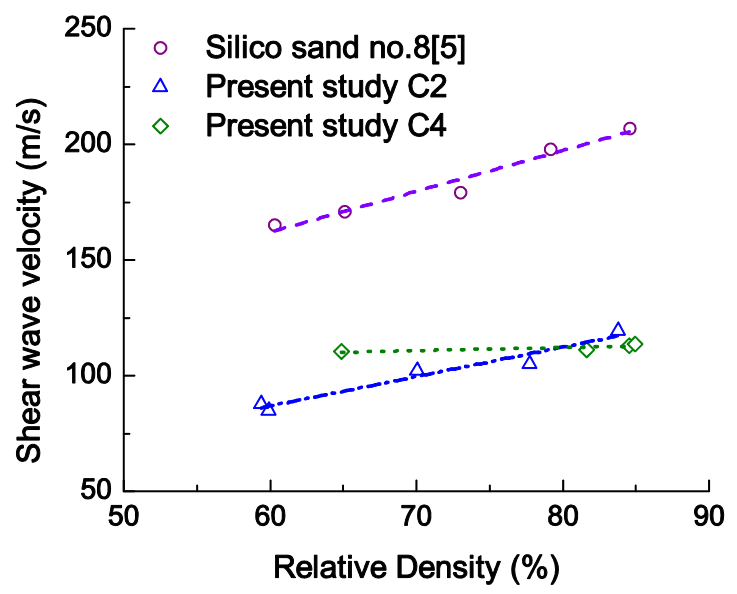

Fig. 4. Quantification of the shear modulus $G_{g}$ $V_{s}-D_{r}$ fitting lines could be expressed as

$$
V_{s}=\mathrm{m} \cdot D_{r}+\mathrm{n}
$$

Numerical shear wave velocity tests assigned shear modulus of $1 \mathrm{GPa}$ to each particles. Doing ratio of the lab test over numerical results on both sides of equation (5) 
could calibrate the shear modulus of Silico sand no.8. PSD curves, C2 and C4, gave dissimilar values of $G_{g}$, as listed in Table 3. It indicates a better gradation would lead to a smaller calibrated value of $G_{g}$.

Table.3 Calibration results of grains' shear modulus by using various PSD curves

\begin{tabular}{lcccr}
\hline PSD curve & $\mathrm{m}$ & $\mathrm{n}$ & $\mathrm{R}^{2}$ & $G_{g}(\mathrm{GPa})$ \\
\hline $\mathrm{C} 2$ & 127.21 & 10.542 & 0.953 & $\mathbf{6 . 0 9 0}$ \\
$\mathrm{C} 4$ & 13.677 & 101.22 & 0.727 & $\mathbf{5 . 1 4 1}$ \\
Xu et al.(2015) & 176.55 & 56.108 & 0.962 & $\mathbf{6 . 6 4 9}$ \\
\hline
\end{tabular}

\subsection{CRR- $V_{s 1}$ curves}

The increase of $C_{u}$ leads to the decreases of calibrated values of both $\mu_{\mathrm{g}}$ and $G_{g}$. The influence of smaller interparticle friction and Shear modulus is converse to the $C R R-V_{\text {s1 }}$ correlation. It shows in single case of $V_{s l}$, from equation (1), lower values of both calibrated parameters have little effect on $C R R$.

Fig. 5 presents the predicted $C R R-V_{s l}$ curves with both calibration results of $\mu_{g}$ and $G_{g}$ and 204 CSR data from the dynamic centrifuge tests[3]. It shows all newly produced curves are very close to the reference curve[4]. Effects of calibrated $\mu_{g}$ and $G_{g}$ could eliminate each other. Although PSD of assemblies affects the calibration of $\mu_{g}$ and $G_{g}$, little on $C R R-V_{s l}$ correlation has be observed.

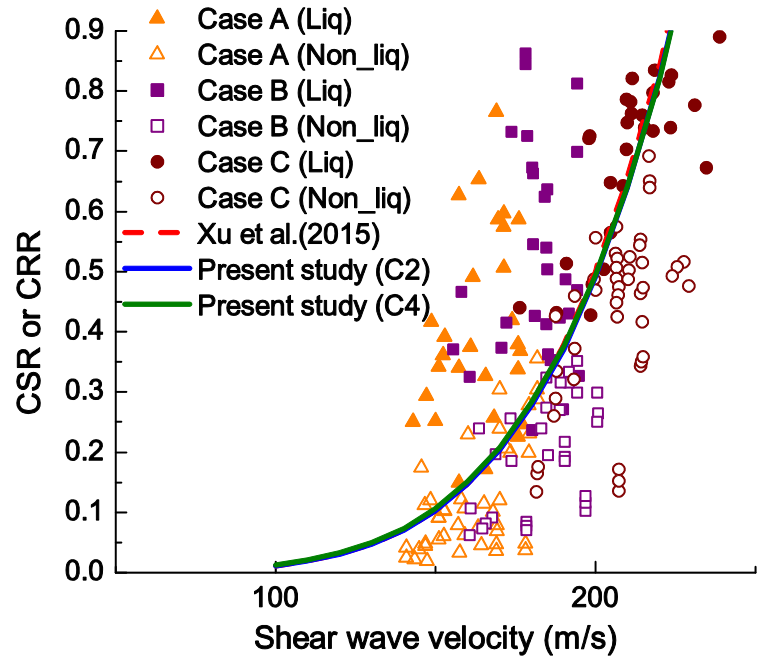

Fig. 5. Super-Influence of calibrated $\mu_{g}$ and $G_{g}$ on $C R R-V_{s I}$ correlation

\section{Conclusions}

By following the systematic parametric method pioneered by previous researches[3, 4, 11, 15], this study has explored the influence of PSDs on correlation between liquefaction resistance and shear wave velocity $\left(C R R-V_{s I}\right)$ of granular soils by performing a series of drained straincontrolled tri-axial tests and shear wave velocity tests, on DEM specimens with various relative density and particle mechanical properties. The findings of this study is that the $C R R-V_{S I}$ correlation is possibly independent of soils' gradation. PSD affects two key parameters' calibrations which determining this correlation. But changes that a new PSD brings to inter-particle friction parameter $\left(\mu_{g}\right.$, and shear modulus $\left(G_{g}\right)$ have converse effects on the eventual $C R R-V_{S I}$ correlation, and eliminate each other. The $C R R-V_{\mathrm{s} 1}$ curve remains still when coefficient of uniformity is increasing.

This study does not consider the breakage nor shape effects of particles. The coefficient of uniformity of 6.0 categorizes soil samples into "well-graded" or "poorlygraded" groups. Both PSD curves employed above have coefficient of uniformity lower than 6.0. Some researches[16-18] on particles crushing within soil samples have shown a tendency that more particles would crush or break in poorly graded soil than in well graded soil under shearing conditions. Spheres were generated as particles in this study, which was meant to isolate effects of contact friction. Spheres tends to undertake more rotation comparing to very angular particles. Assemblies of long or angular particles in DEM simulation, show behaviours closer to the practice while bearing shearing[15, 19].

\section{References}

1. Y. Zhou, Y. Chen, J Geotech Geoenvironmental Eng 133, 959-972. (2007)

2. Y. Chen, Y. Zhou, H. Ke, 14th World Conf Earthq Eng, 1-8 (2008)

3. Y. Zhou, Y. Chen, Y. Shamoto, J Geotech Geoenvironmental Eng 136,165-177 (2010)

4. X. Xu, D. Ling, Y. Cheng, Y. Chen, Geotechnique 65, 337-348 (2015)

5. $\mathrm{X}$. $\mathrm{Xu}$, Study on the micromechanism of sand liquefaction and its evaluation, Zhejiang University (2012) (not published)

6. C. Thornton, Geotechnique, 50, 43-53 (2000)

7. X. Huang, K.J. Hanley, C. O'Sullivan, F.C.Y. Kwok Particuology 15, 107-115 (2014)

8. K. Soga, S. Yimsiri, Géotechnique 60, 483-495 (2010)

9. E.M. Kara, Eng Technol Appl Sci Res 3, 497-501 (2013)

10. A. Hamidi, E, Azini, B. Masoudi, Sci Iran 19, 393 402 (2012)

11. X. Xu, Y. Cheng, D. Ling, AIP Conf. Proc., 201-204 (2013)

12. Itasca, Manual of particle flow code in 3-dimension version 3.00 (2003)

13. C. Salot, P. Gotteland, P. Villard, Granul Matter 11, 221-236 (2009)

14. J.C. Lopera Perez, C.Y. Kwok, C. O’Sullivan, X. Huang, K.J. Hanley, Géotechnique 66, 725-740 (2016)

15. Y. Yu, Y. Cheng, X. Xu, K. Soga, Geomechanics from Micro to Macro, 207-212 (2015)

16. M.R. Coop, F.N. Altuhafi, Géotechnique 61, 459471. (2011)

17. F.N. Altuhafi, B. Baudet, P. Sammonds, Can Geotech J 790, 775-790 (2010)

18. F. Altuhafi, B.A. Baudet, Eng Geol 120, 1-9 (2011)

19. R.P. Jensen, P.J. Bosscher, M.E. Plesha, T.B. Edil, Int J Numer Anal Methods Geomech 23, 531-547 (1999) 